\title{
Topology optimization for optical microlithography with partially coherent illumination
}

\section{Zhou, Mingdong; Lazarov, Boyan Stefanov; Sigmund, Ole}

\section{Published in:}

International Journal for Numerical Methods in Engineering

Link to article, DOI:

10.1002/nme.5299

Publication date:

2017

Document Version

Peer reviewed version

Link back to DTU Orbit

Citation (APA):

Zhou, M., Lazarov, B. S., \& Sigmund, O. (2017). Topology optimization for optical microlithography with partially coherent illumination. International Journal for Numerical Methods in Engineering, 109(5), 631-647. https://doi.org/10.1002/nme.5299

\section{General rights}

Copyright and moral rights for the publications made accessible in the public portal are retained by the authors and/or other copyright owners and it is a condition of accessing publications that users recognise and abide by the legal requirements associated with these rights.

- Users may download and print one copy of any publication from the public portal for the purpose of private study or research.

- You may not further distribute the material or use it for any profit-making activity or commercial gain

- You may freely distribute the URL identifying the publication in the public portal 


\title{
Topology optimization for optical microlithography with partially coherent illumination
}

\author{
Mingdong Zhou*†, Boyan S. Lazarov and Ole Sigmund \\ Section of Solid Mechanics, Department of Mechanical Engineering, Technical University of Denmark, Nils Koppels \\ Allé, Building 404, DK-2800 Kgs. Lyngby, Denmark
}

\begin{abstract}
SUMMARY
This article revisits a topology optimization design approach for micro-manufacturing and extends it to optical microlithography with partially coherent illumination. The solution is based on a combination of two technologies, the topology optimization and the proximity error correction in micro-/nano- lithography. The key steps include (i) modeling the physical inputs of the fabrication process, including the UV-light illumination source and the mask, as the design variables in optimization and (ii) applying physical filtering and heaviside projection for topology optimization, which correspond to the aerial image formulation and the pattern development processes, respectively. The proposed approach results in an effective source and a binary design mask, which can be sent directly to fabrication without additional post-processing steps for proximity error correction. Meanwhile, the performance of the device is optimized and robust w.r.t. process variations, such as dose / photo-resist variations and lens defocus. A compliant micro-gripper design example is considered to demonstrate the applicability of this approach. CCC
\end{abstract}

Received ...

KEY WORDS: Topology Optimization; Optical Microlithography; Partially Coherent Illumination; Robust Source and Mask Optimization; MEMS

\section{INTRODUCTION}

The focus of this paper is on improving the topology-optimization design approach for manufacturing with microlithography $[1,2]$ and extending it to Source and Mask Optimization (SMO) for Optical Projection Lithography (OPL) with a partially coherent imaging model. The solution is based on a combination of the topology optimization method for mechanical design and the proximity error correction (PEC) approach in computational lithography. The optimization results comprise an effective illumination source and a binary mask, which can be sent to manufacturing without additional PEC. Simultaneously, the printed pattern on the wafer possesses an optimized and robust functionality w.r.t. process variations.

\subsection{Optical Projection Lithography and Proximity Error Correction}

OPL is a micro- / nano- manufacturing process for fabricating Integrated Circuits (IC), LightEmitting Diode (LED) and MicroElectroMechanical Systems (MEMS). It utilizes UV-light to transfer the design patterns (also known as the blueprint), which are originally depicted on a mask, onto a silicon wafer. The process is schematically illustrated in Fig. 1. The UV-light from an illumination source passes through a set of optical systems, as well as a mask, and it is eventually

*Correspondence to: Mingdong Zhou, Building 404, Nils Koppels Allé, DK-2800 Kgs. Lyngby, Denmark
${ }^{\dagger}$ Email:minzho@mek.dtu.dk

$\mathrm{CCC}$

$X X X$ 


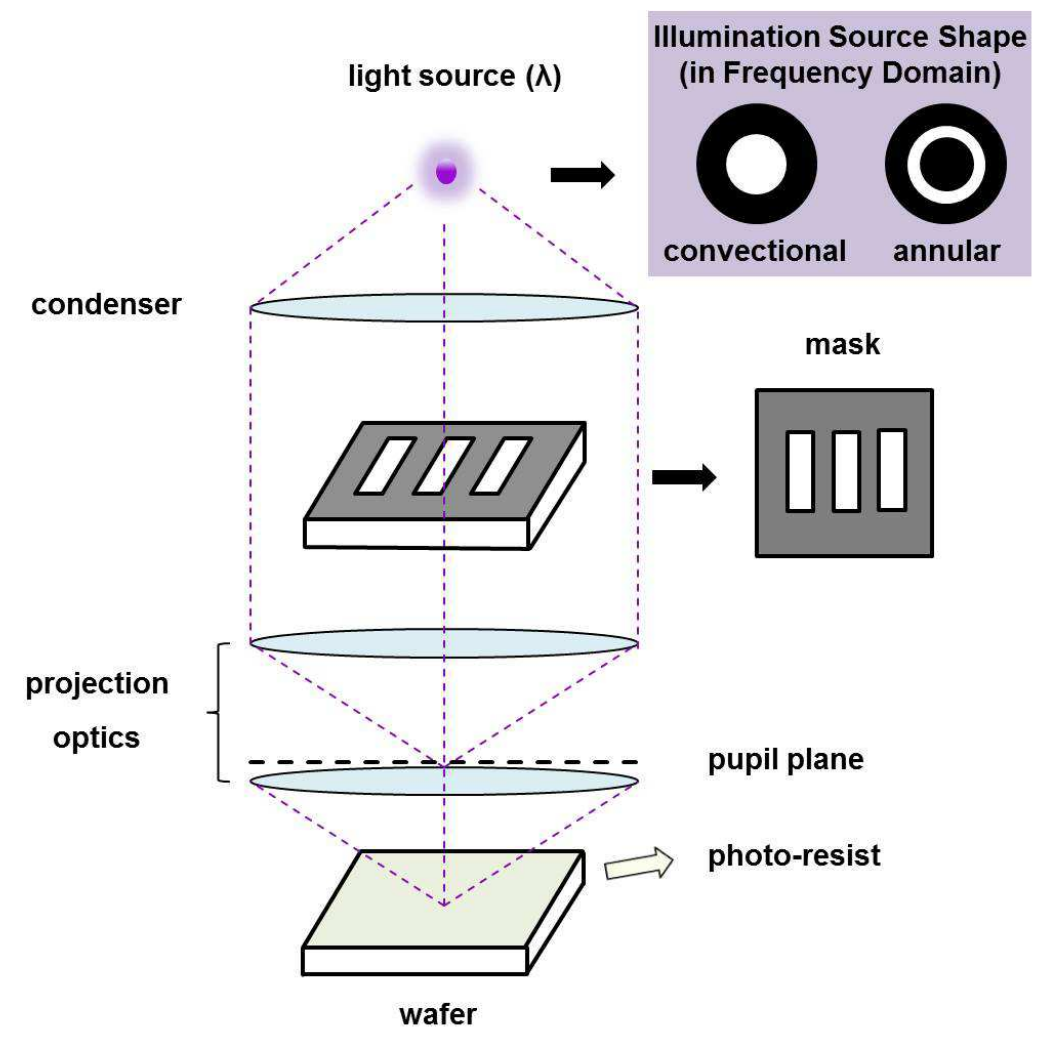

Figure 1. Optical projection lithography with partially coherent illumination. The shape of illumination source is plotted in spatial frequency coordinates, representing the image of the actual source in the pupil plane.

projected down onto a Photo-Resist (PR) coated wafer. The planar mask contains transparent and opaque layouts to represent the blueprint. The transparent regions allow UV-light to pass through and result in a diffracted light pattern that resembles the blueprint. The light is then collected by the projection optical lens and reaches the wafer. The PR is sensitive to photons and the exposed $\mathrm{PR}$ regions can be removed (positive resists) or retained (negative resists) in a development process. As a result, a resist pattern remains on the wafer and may be subject to further post-processing, e.g. pattern transfer. However, due to the diffractive nature of light and the inherent limitations (e.g. limited lens size) of the optical systems, the developed pattern usually exhibits a distorted version of the blueprint and tiny features with size below a certain resolution limit may vanish. In reality, such a loss of pattern fidelity leads to short circuits and yield loss for IC applications or malfunctioning MEMS structural layouts. In order to improve the resolution and fidelity of micro- / nano- devices, various Resolution Enhancement Techniques (RET) as reviewed in [3] are applied, among which the Proximity Error Correction (PEC) has been extensively investigated and applied.

PEC is a set of mathematical approaches aiming at compensating the process errors due to the inherent diffraction nature of OPL and the electron scattering phenomenon in Electronic Beam Lithography (EBL). The methods range from empirically rule-based to optimization-based algorithms. For EBL, one rule-based algorithm is the PROLYTH by [4], which resorts to predefined rules to correct the input electron dosage in a hierarchical way. The optimization-based approaches $[5,6,7]$ require a larger computational effort by solving an inverse design problem for finding optimized electron dosage such that the physical realization is as close as possible to the blueprint design. Both approaches require a nonlinear modeling of the EBL process. For OPL, various RETs $[3,8,9]$ have been proposed by modulating the input mask [10] or the illumination source [11, 12] 
in order to ensure patterns of the best quality. Other methods include utilization of phase-shift mask [13] and off-axis illuminations [8].

Another advanced PEC solution, namely Source and Mask Optimization (SMO), has recently received extensive interest. The idea of SMO is to find an optimized pair of mask and illumination source that minimizes the discrepancy between the developed pattern and the blueprint. Hence, it allows for more design freedom than the sole mask or source optimization for PEC. This approach was first proposed by [14] and then followed by research works on the pixelated gradient-based SMO algorithms [15, 16, 17, 18, 19, 20, 21, 22, 23]. A variety of computational workflows such as simultaneous, sequential and hybrid SMO schemes has been proposed to solve the optimization problem [22]. On the manufacturing side, a parallel progress on the fabrication technology based on a programmable mirror array [24] facilitates practical applications of SMO solutions.

\subsection{Combining Proximity Error Correction with Topology Optimization}

In the area of mechanical engineering, topology optimization $[25,26]$ is a well-established methodology, which initially was proposed for designing weight efficient structures and now is being applied to multi-disciplinary problems, such as to design of MEMS [27] and photonic crystal waveguide [28]. It is regarded as a generative design tool utilizing computer simulation and optimization techniques, compared to conventional means based on designer's experience and intuitions. In topology optimization, the design problem is interpreted as an optimal materialdistribution problem over a predefined domain. The optimized topology is calculated iteratively based on a numerical simulation of the considered physics.

The 2D topology optimization shares similarities to PEC in the model parameterizations (a pixelized image) and the computational workflow [1]. On one hand, the three-field topology optimization scheme consists of a design, an intermediate and a material density field. They are correlated through mathematical smoothing and heaviside projection operations in a sequential order $[29,30,31]$. On the other hand, simulation of EBL and OPL utilize a three-phase parameterizations to model the electron scattering for EBL or UV-light exposure for OPL and etching processes. By identifying such an analogy, these two technologies can be combined into a unified solution for designing optimal devices for micro- / nano- lithography. Specifically, it consists of (i) modeling the physical entities in the fabrication process as the design variables in optimization and (ii) leveraging physical filtering and heaviside projection in topology optimization, which correspond to manufacturing characteristics. The solution was first proposed to design optimized mechanical structures for EBL [1], where the electron dosage and exposure fields in EBL are represented by the design and intermediate fields in topology optimization via an electron scattering model. In the meantime, the material density field from the etching process denotes the structural layout and is used for the physical performance simulation using finite element analysis. The idea was later extended to OPL for mask optimization under spatially coherent illumination [2].

The unified approach is able to design manufacturable micro-/nano-devices and simultaneously ensure a robust functionality. The main advantage is that it reduces the risk of design failure compared to a conventional procedure that one first designs a device and then performs PEC on the manufacturing site. However, real-world photo-lithography uses a (spatially) partial coherent imaging system, in which the illumination is composed of light coming from a range of angles, as oppose to the coherent illumination with only a plane light wave normally incident to the mask [8]. Hence in this paper, the unified approach is extended to source and mask optimization for optical projection lithography with partially coherent illumination. Such an extension is non-trivial, as it introduces a highly non-linear filter and thereby requires a more complex sensitivity analysis as well as an efficient optimization scheme. Manufacturing uncertainties, including lens defocus and dose exposure variations, are considered here through a stochastic formulation of the actual pattern behavior.

The remainder of this paper is organized as follows. Section 2 introduces the mathematical modeling of the optical projection lithography process under partially coherent illumination and SMO. Section 3 reviews the three-field topology optimization scheme. The unified solution is 


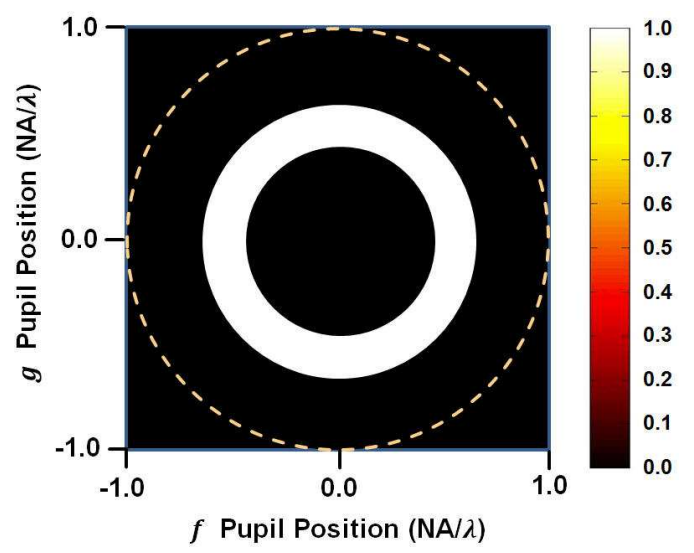

(a) Effective illumination source

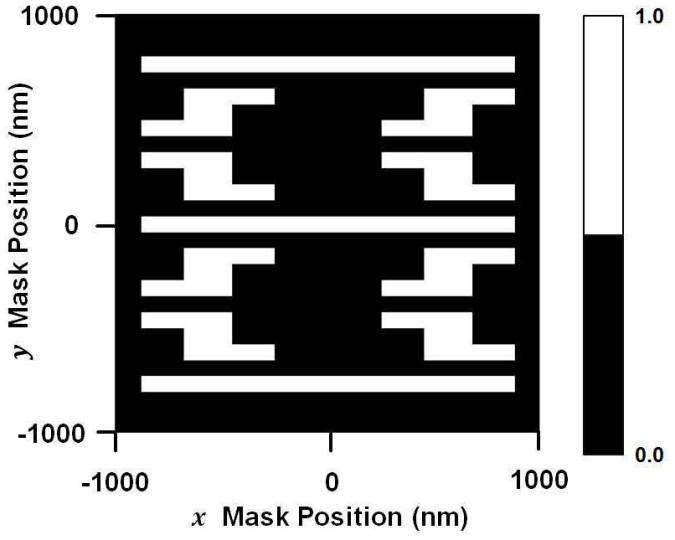

(b) Mask

Figure 2. Partially coherent UV-light source and binary mask: (a) an annular effective light source (plotted in spatial frequency coordinate), where the dashed circle represents the cutoff frequency NA/ $\lambda$ of the circular pupil and the color-bar denotes the normalized light intensity; (b) a binary mask containing an IC blueprint.

presented in Section 4 and it is followed by a compliant micro-gripper design example in Section 5. Possible extensions of the solution are discussed in Section 6.

\section{SOURCE AND MASK OPTIMIZATION IN OPTICAL PROJECTION LITHOGRAPHY}

\subsection{Simulation of OPL process with partially coherent illumination}

In an OPL system as shown in Fig. 1, a (monochromatic) UV-light source placed at the focal point of the condenser lens creates an image, known as the effective light source, in the pupil plane. The effective source represents the composite spectrum of all illumination light rays in the absence of the mask [3]. In practice, it is common to mathematically formulate the OPL process and PEC based on this effective source, which in turn can be physically realizable with a programmable mirror array [24]. Fig. 2(a) illustrates an example of an annular effective source plotted in spatial frequency coordinate which has full light intensity in the annular region. Each point on this image corresponds to one spatially coherent illumination producing a plane wave striking the mask at a certain angle. The (normalized) intensity of each plane wave can be designed continuously from 0.0 to 1.0. Different point sources do not interact coherently with each other. Note that for a coherent illumination normally incident to the mask, the source shape degenerates to a point at the origin of the frequency domain. Hence, considering the partially coherent illumination in optimization allows for more design freedom from the source comparing to the previous work with a coherent illumination [2]. The dashed circle (brown color) shown in Fig. 2(a) is centered at the origin $(f, g)=(0,0)$ with a radius equal to the cutoff frequency NA/ $\lambda$ of the lens, where $\lambda$ is the wavelength of the light and NA is the numerical aperture of the lens. For the source and mask optimization described in later sections, the design domain of the source is confined inside such a circular region as the outlier does not contribute to the arial-image formation. However, the design domain of the mask is the overall discretized image as shown in Fig. 2(b) for an IC pattern example.

The OPL process transfers a design pattern on a mask to a photoresist-coated wafer via partially coherent UV-light. Mathematically, it can be modeled in two consecutive steps: (i) UV-light exposure and (ii) photoresist development [3]:

$$
P(\mathbf{x})=\Gamma(A(\phi(\mathbf{x}), \boldsymbol{\sigma}(\mathbf{w}))),
$$

where $P$ denotes the output pattern on the wafer, $\Gamma$ represents the process of photoresist development and $A$ is the arial image representing the light intensity projected onto the photo-resist. The input 


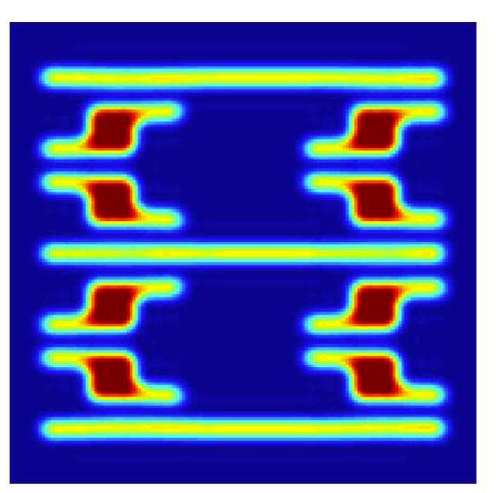

(a) Aerial image
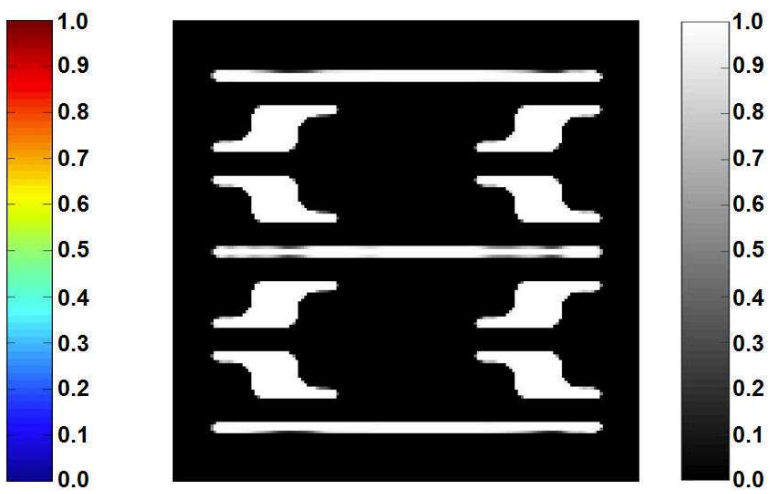

(b) Developed pattern

Figure 3. OPL simulation using the source and mask shown in Fig. 2 as the input: (a) aerial image, where the color scale represents the (normalized) light intensity projected onto the wafer; (b) developed pattern with pattern error $\mathrm{PE}=34.1 \%$. (N.B. The color scale denotes structural density distribution for numerical examples in Sec. 5).

binary mask $\phi(\mathbf{x})$ has the value of 1 for a transparent pixel and 0 for an opaque one. The normalized intensity of the illumination source $\boldsymbol{\sigma}(\mathbf{w})$ is continuous $\boldsymbol{\sigma} \in[0,1]$. Here, $\mathbf{x}=(x, y)$ is the spatial coordinate of the mask and $\mathbf{w}=(f, g)$ is the frequency coordinate of the effective source.

The aerial image is obtained using Abbe's method of partially coherent image calculation [32] by first calculating the coherent aerial images from the each discrete point on the source and then summing up all the intensity contributions:

$$
A(\mathbf{x})=\frac{\sum_{i=1}^{N_{s}} \sigma_{i}\left[\left(\phi \otimes H_{i}\right)(\mathbf{x})\right]^{2}}{\sum_{i=1}^{N_{s}} \sigma_{i}\left\|\Sigma_{\mathbf{x}} H_{i}(\mathbf{x})\right\|^{2}},
$$

where $N_{s}$ is the total number of discretized source points in the frequency domain and $H_{i}$ denotes the point spread function (PSF) ${ }^{\dagger}$ associated to each source point at $\left(f_{i}, g_{i}\right)$. The intensity of the aerial image is normalized by the image intensity of using a transparent mask without any feature. For a circular lens pupil with a cutoff frequency of NA/ $\lambda$, the PSF $H_{i}$ is defined using Zernike polynomials [8] as:

$$
\begin{gathered}
H_{i}(\mathbf{x})=\mathrm{IFT}^{-1}\left\{h_{\beta}(\mathbf{w})\right\} \cdot \exp \left\{-j 2 \pi\left(f_{i} x+g_{i} y\right)\right\}, \\
h_{\beta}(\mathbf{w})=h(\mathbf{w}) \cdot \exp \left\{j \pi \beta\left(\lambda|\mathbf{w}|^{2}-\frac{\mathrm{NA}^{2}}{2 \lambda}\right)\right\}, \\
h(\mathbf{w})=\left\{\begin{array}{lc}
1, & \text { if }\|\mathbf{w}\| \leq \mathrm{NA} / \lambda \\
0, & \text { otherwise }
\end{array}\right.
\end{gathered}
$$

where $\otimes$ stands for the convolution operator, IFT ${ }^{-1}$ denotes the inverse Fourier transform, $\beta$ is the defocus distance between the actual imaging plane and the focal plane and $\|\bullet\|$ is the Euclidean norm.

The etching process on the photoresist is simulated using an approximated Heaviside step function:

$$
P(\mathbf{x})=\frac{\tanh (\alpha \cdot \eta)+\tanh (\alpha \cdot(A(\mathbf{x})-\eta))}{\tanh (\alpha \cdot \eta)+\tanh (\alpha \cdot(1.0-\eta))},
$$

\footnotetext{
${ }^{\dagger}$ The discussion in this paper is based on $1 \mathrm{x}$ imaging projection, where the dimension of the mask is the same as the pattern being produced. A reduction imaging (e.g. $4 \mathrm{x}$ ) system allows for an easier fabrication of the mask in practice due to the increased size of the mask. However, imaging with reduction is mathematically treated the same way as for $1 \mathrm{x}$ system, except that a radiometric correction term should be added to the PSF [8].
} 
where $\alpha$ controls the sensitivity of the photoresist and $\eta$ defines the exposure dose threshold. This function shares a similar profile to the sigmoid function $1 /\left(1+e^{-\alpha(A(\mathbf{x})-\eta)}\right)$ for a large $\alpha$ value (e.g. $\alpha>32$ ) but it interpolates the interval $[0,1]$ when a small $\alpha$ value is used (e.g. $\alpha<8$ ) differently. The latter property is crucial in ensuring a successful continuation-based optimization process, as previously studied in [2]. The profile of the approximated Heaviside step function for different $\alpha$ values is given in Fig. 4.

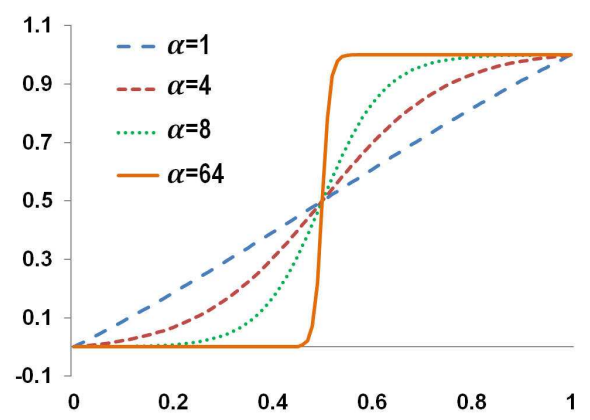

Figure 4. The profile of the heaviside function Eq. 6 for different $\alpha$ values.

Fig. 3 demonstrates one simulation result of the OPL process described by Eqns. (1-6). In this example, the annular source and the IC-pattern shown in Fig. 2 are used as the inputs. The process is assumed to be under the ideal manufacturing condition with a photoresisit threshold of $\eta=0.5$ and a defocus value of $\beta=0 \mathrm{~nm}$. The source and the mask are discretized into $101 \times 101$ and $160 \times 160$ pixels, respectively. The system parameters are set as follows: $\lambda=193 \mathrm{~nm}, N A=1.25$ and $\alpha=64$. The final developed pattern shown in Fig. 3(b) deviated from the blueprint $P_{0}$ by a Pattern Error of $\mathrm{PE}=34.1 \%$, where $\mathrm{PE}$ is defined as:

$$
\mathrm{PE}=\frac{\left\|\mathrm{P}(\mathbf{x})-\mathrm{P}_{0}(\mathbf{x})\right\|}{\mathrm{P}_{0}(\mathbf{x})} .
$$

\subsection{Source and mask optimization}

The goal of PEC is to solve an inverse problem on the manufacturing site in order to find a pair of source and mask, which can reduce the pattern error between the printed pattern $P$ and the blueprint $P_{0}$. The standard discrete PEC problem is given as follows:

$$
\begin{aligned}
\min _{\boldsymbol{\phi}, \boldsymbol{\sigma}}: & J(\boldsymbol{\phi}, \boldsymbol{\sigma})=\frac{1}{N_{m}}\left\|P(\mathbf{x})-P_{0}(\mathbf{x})\right\|, \\
\text { s.t. : } & \phi_{i}=\{0,1\}, \quad i=1: N_{m}, \\
: & 0 \leq \sigma_{j} \leq 1, \quad j=1: N_{s},
\end{aligned}
$$

where $N_{m}$ is the total number of pixels in the mask. This optimization problem can be simplified and solved in various ways for practical considerations of computation and manufacturing.

Firstly, the binary mask $\phi_{i}=\{0,1\}$ is modeled using a continuous variable $\phi_{i} \in[0,1]$ in the optimization in order to circumvent the challenge in large-scale integer programming. A typical way to steer the solution to a discrete results is by adding some forms of penalty $[33,2]$ to the objective function. Secondly, manufacturing uncertainties are ubiquitous in practical fabrication, such as mechanical fluctuation, lens defocus, dose variation or inhomogeneous photoresist coating. However, the inverse problem Eq. (8) together with Eqs. (1-6) are only effective in finding optimized source and mask for a certain manufacturing condition, e.g. a fixed lens defocus $\beta$ and a photoresist threshold $\eta$. When a range of process variation is considered, a stochastic $[34,18,35,33,21,36]$ or a worst-case [37,2] formulation must be used to account for manufacturing uncertainties and to ensure a robust device performance. Moreover, the objective functional can be augmented with different penalty terms for source and mask regularizations, such as to manipulate the pupil fill rate to enhance 
the process window [38], to control the minimum integrated pupil fill percentage and minimum dark pixel intensity to ensure the source manufacturability [22] as well as to reduce the mask complexity for cost- and time-efficient mask fabrication [39]. The overall SMO problem of Eq. 8 can be solved with either simultaneous, sequential or hybrid optimization schemes. The simultaneous scheme co-optimizes the source and mask variables at the same time in order to fully utilize their interaction. However, the sequential scheme alternates the mask and source optimizations, which is computationally more efficient and allows for more flexibility to control the optimization workflow. The hybrid scheme combines the previous two and exhibits improvement on specific IC examples [22]. Interested readers are referred to the study in [22] and references therein for more discussion.

\section{THREE-FIELD TOPOLOGY OPTIMIZATION}

Topology optimization addresses the optimal-material-distribution problem by minimizing an objective function $C$, subject to various constraints, such as governing physics equations, volume, geometric restrictions, etc. A general disretized formulation of a topology optimization problem using a three-field scheme $[29,30,37]$ is given as:

$$
\begin{aligned}
\underset{\boldsymbol{\rho}}{\min .:} & C(\overline{\boldsymbol{\rho}}, \boldsymbol{u}(\overline{\boldsymbol{\rho}})), \\
\text { s.t. : } & R(\overline{\boldsymbol{\rho}}, \boldsymbol{u}(\overline{\boldsymbol{\rho}}))=0, \\
: & G_{i}(\overline{\boldsymbol{\rho}}, \boldsymbol{u}(\overline{\boldsymbol{\rho}})) \leq 0, \quad i=1, \ldots, n,
\end{aligned}
$$

where $\boldsymbol{\rho}$ is the design variable vector, $\bar{\rho}$ denotes the material density, $\mathbf{u}$ is the state variable satisfying a linear or non-linear state equation $R$ and the list of $G_{i}$ are the other constraints. The design variable field $\rho$ and the material density field $\bar{\rho}$ are related through filtering-threshold operations via a third intermediate field $\tilde{\rho}$ :

$$
\begin{gathered}
\tilde{\rho}_{i}=\frac{\sum_{j \in N_{i}} \omega\left(\mathbf{x}_{j}\right) v_{j} \rho_{j}}{\sum_{j \in N_{i}} \omega\left(\mathbf{x}_{j}\right) v_{j}}, \quad \omega\left(\mathbf{x}_{j}\right)=r-\left|\mathbf{x}_{i}-\mathbf{x}_{j}\right|, \\
\bar{\rho}_{i}=\frac{\tanh (\alpha \cdot \eta)+\tanh \left(\alpha \cdot\left(\tilde{\rho}_{i}-\eta\right)\right)}{\tanh (\alpha \cdot \eta)+\tanh (\alpha \cdot(1.0-\eta))},
\end{gathered}
$$

where $\boldsymbol{N}_{i}$ represents the neighborhood element set of the element $i, r$ is the filter radius, $v_{j}$ is the volume of the element $j$ and $\omega$ is a weighting function of the distance between the central coordinate of cell $i$ and $j$. The heaviside projection function Eq. (11), which is used to ensure a solid-void structural layout by applying a large $\alpha$ value (e.g. $\alpha \geq 32$ ). Note that, if only the filtering operation Eq. (10) is used in topology optimization, it regularizes the optimization problem [40] and ensures a mesh-independent design process, e.g. the optimized result will exhibit a minimum length scale. But with the additional projection operation Eq. (11), the regularization effect is destroyed and no minimum length can be guaranteed [37]. However, as will be seen in later sections, using a robust formulation is able to impose a finite minimum length scale to the optimized design, as it also has been proven for the worst-case based robust formulation [37].

This three-field topology optimization scheme, consisting of the fields $\rho, \tilde{\rho}$ and $\bar{\rho}$, respectively, shares similarity to OPC in computational lithography, for which the process simulation also consists of three correlated stages, namely the mask and source (as the input), the aerial image and the developed pattern (as the output). Hence, it offers the possibility to combine these two computational design approaches into a manufacturing-aware topology optimization solution.

\section{A UNIFIED DESIGN SOLUTION FOR OPTICAL MICROLITHOGRAPHY}

In a conventional workflow of first designing a blueprint and then performing PEC, the two separate activities focus on different criteria, the device performance and the manufacturability, respectively. 


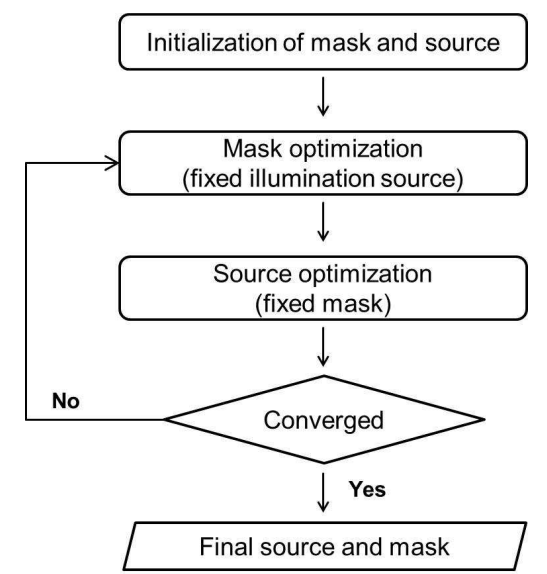

Figure 5. A sequential scheme for source and mask optimization.

In such a case, the performance of the final device can be degraded due to the fact that OPC does not consider the functionality during the optimization and it is unable to replicate the blueprint if the feature resolution is lower than the resolution limit.

In order to ensure a consistent functionality of the blueprint and the final pattern, a unified design solution for optical micro-lithography is proposed here. The approach directly takes the device performance into account in the problem formulation and replaces the field variables in topology optimization with those of computational lithography, namely $\rho$ to $\phi$ (input mask) and $\sigma$ (illumination source), $\tilde{\boldsymbol{\rho}}$ to $A$ (arial image) and $\bar{\rho}$ to $P$ (physical structure) $)^{\ddagger}$. From the perspective of topology optimization, the design fields now are the mask and source; the aerial image becomes the intermediate filtered field; and the developed pattern represents the projected structural layout. The new fields $\phi, \sigma, A$ and $P$ are related through the physical manufacturing process model given by Eqn. (1-6), rather than mathematical regularization in topology optimization. Process uncertainties of lens defocus and exposure dose variation (photo-resist variation) are considered here using a risk-neutral stochastic formulation similar to that in $[35,33,21]$. Hence, the overall topology optimization problem for optical projection lithography becomes:

$$
\begin{aligned}
\underset{\phi, \sigma}{\min .} & L=E\left\{C_{\eta}^{\beta}\right\}, \\
\text { s.t. : } & R\left(P_{\eta}^{\beta}, \mathbf{u}_{\eta}^{\beta}\right)=0, \\
: & G_{i}\left(P_{\eta}^{\beta}, \mathbf{u}_{\eta}^{\beta}\right) \leq 0, \quad i=1, \ldots, n, \\
: & \beta \in\left[\beta_{\min }, \beta_{\max }\right], \eta \in\left[\eta_{\min }, \eta_{\max }\right], \\
: & \phi_{i}=\{0,1\}, \quad i=1: N_{m}, \\
: & 0 \leq \sigma_{j} \leq 1, \quad j=1: N_{s} .
\end{aligned}
$$

In this formulation, $P_{\eta}^{\beta}$ denotes pattern developed at the lens defocus $\beta$ and exposure threshold $\eta$. $C_{\eta}^{\beta}$ and $\mathbf{u}_{\eta}^{\beta}$ are the corresponding objective value and the state variable evaluated upon $P_{\eta}^{\beta}$, respectively. Furthermore, $E$ captures the mean objective value over the variation ranges $\left[\beta_{\min }, \beta_{\max }\right]$ for the lens defocus and $\left[\eta_{\min }, \eta_{\max }\right]$ for the threshold. In practice, several sampling patterns developed with different pairs of $[\beta, \eta]$ are used to calculate the mean value. Note that this formulation only minimizes the mean performance of an ensemble of possible realizations without imposing any restrictions on the spread.

\footnotetext{
${ }^{\ddagger}$ For simplicity, an ideal pattern transfer is assumed in the current work, so that the final structural layout is the same as the developed pattern. Including the pattern transfer process and associated further errors can potentially be subject of further research and can probably be included in the topology optimization process as well.
} 
The proposed problem can be solved efficiently under a sequential SMO scheme [16, 22] as illustrated in Fig. 5. Upon initializing a pair of source and mask, the mask is first optimized under the fixed illumination source and then the source is updated and optimized w.r.t. the obtained mask. The process iterates until some convergence criteria are satisfied or the iteration count reaches a predefined limit.

In the mask optimization, the illumination source is fixed and the discrete problem $\phi=\{0,1\}$ is solved using a continuous formulation as:

$$
\begin{aligned}
\min _{\phi}: & L=E\left\{C_{\eta}^{\beta}\right\}+\frac{\lambda_{1}}{n_{e}} \sum_{e=1}^{n_{e}} \phi\left(\mathbf{x}_{e}\right)\left(1-\phi\left(\mathbf{x}_{e}\right)\right)+\frac{\lambda_{2}}{n_{e}} \sum_{e=1}^{n_{e}}\left|\nabla \rho\left(\mathbf{x}_{e}\right)\right|^{2}, \\
\text { s.t. : } & R\left(P_{\eta}^{\beta}, \mathbf{u}_{\eta}^{\beta}\right)=0, \\
: & G_{i}\left(P_{\eta}^{\beta}, \mathbf{u}_{\eta}^{\beta}\right) \leq 0, \quad i=1, \ldots, n, \\
: & \beta \in\left[\beta_{\min }, \beta_{\max }\right], \eta \in\left[\eta_{\min }, \eta_{\max }\right], \\
: & 0 \leq \phi(\mathbf{x}) \leq 1 .
\end{aligned}
$$

Two penalty terms with user-defined weights $\lambda_{1}$ and $\lambda_{2}$ are added to the objective functional to penalize the intermediate pixel value and suppress the noise in the mask, respectively. The aerial image is calculated with a reduced basis approach [41]:

$$
A(\mathbf{x})=\frac{\sum_{i=1}^{N_{s}} \sigma_{i}\left[\left(\phi \otimes H_{i}\right)(\mathbf{x})\right]^{2}}{\sum_{i=1}^{N_{s}} \sigma_{i}|| \Sigma_{\mathbf{x}} H_{i}(\mathbf{x}) \|^{2}} \approx \frac{\Sigma_{j=1}^{M_{s}}\left[\left(\phi \otimes G_{j}\right)(\mathbf{x})\right]^{2}}{\sum_{i=1}^{N_{s}} \sigma_{i}\left\|\Sigma_{\mathbf{x}} H_{i}(\mathbf{x})\right\|^{2}},
$$

where the new PSF set $G$ (with total number $M_{s}<<N_{s}$ ) helps to gain tremendous speed-up in each design iteration with acceptable accuracy. In the source optimization, the binary mask obtained from the previous optimization is used as the fixed input and the full Abbe's model Eq. (2) is used to compute the aerial image. The optimization problem is written as:

$$
\begin{aligned}
\underset{\sigma}{\min .:} & L=E\left\{C_{\eta}^{\beta}\right\} \\
\text { s.t. : } & R\left(P_{\eta}^{\beta}, \mathbf{u}_{\eta}^{\beta}\right)=0, \\
: & G_{i}\left(P_{\eta}^{\beta}, \mathbf{u}_{\eta}^{\beta}\right) \leq 0, \quad i=1, \ldots, n, \\
: & \beta \in\left[\beta_{\min }, \beta_{\max }\right], \eta \in\left[\eta_{\min }, \eta_{\max }\right], \\
: & 0 \leq \sigma(\mathbf{x}) \leq 1 .
\end{aligned}
$$

Comparing to a simultaneously SMO scheme [22], which directly uses the full Abbe's model Eq. (2) to calculate the aerial image during the optimization process, the main benefit of using the sequential SMO scheme is lower computational efforts. The gain comes from two aspects: (i) by using the reduced basis approach Eq. (14) in calculating the areal image when the source is fixed in the mask optimization and (ii) by pre-computing and reusing the value of $\left[\left(\phi \otimes H_{i}\right)(\mathbf{x})\right]$ and $\left\|H_{i}(\mathbf{x})\right\|^{2}$ for the arial- image calculation Eq. (2) and sensitivity analysis Eq. (23) in the source optimization, provided that the mask is fixed.

\section{A COMPLIANT MICRO-GRIPPER DESIGN EXAMPLE}

\subsection{Design problem}

This section studies a benchmark topology optimization example, designing a compliant microgripper [27, 42], to demonstrate the applicability of the proposed solution.

Fig. 6(a) illustrates the design domain $\Omega$ (blue color) and the boundary conditions. On the left side, two corners are clamped and an input force exerted at the middle. The force is modeled as a strain-based actuator defined by blocking force $f_{i n}$ and a spring of stiffness $k_{i n}$ [27]. On the right side, two tips of the jaws (solid black region) are considered as the output, where two springs of 


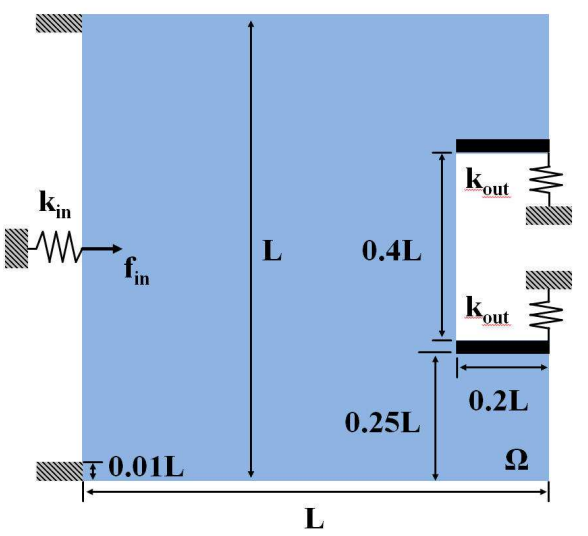

(a)

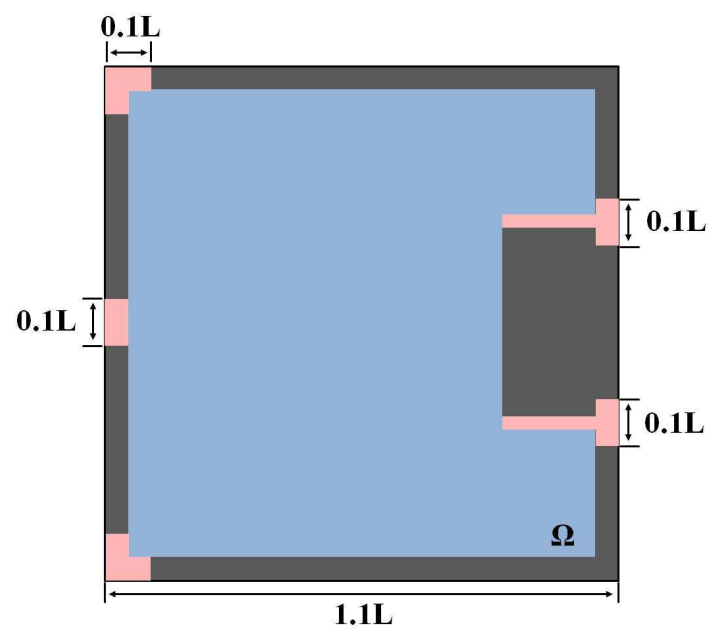

(b)

Figure 6. Topology optimization of a compliant micro-gripper: (a) the design domain $\Omega$ and boundary conditions; (b) the initial mask containing $\Omega$, opaque non-design domain (dark) and transparent supportive features (red).

stiffness $k_{\text {out }}$ are attached to simulate the reaction with the target object. The objective is to design a micro-gripper inside $\Omega$ which can produce maximized vertical displacement at the outputs under a structural volume constraint. By discretizing the design domain $\Omega$ into a finite element mesh, the design problem is formulated into an optimal material-distribution problem as:

$$
\begin{array}{cl}
\underset{\boldsymbol{\rho}}{\min }: & C(\boldsymbol{\rho})=-\mathbf{l}^{\top} \mathbf{u}, \\
\text { s.t. : } & \mathbf{K}(\overline{\boldsymbol{\rho}}) \mathbf{u}=\mathbf{f}, \\
: & \overline{\boldsymbol{\rho}}^{\top} \mathbf{v} \leq V^{*}, \\
: & \mathbf{0} \leq \boldsymbol{\rho} \leq \mathbf{1},
\end{array}
$$

where $C$ is the objective functional, $\mathbf{u}$ is the displacement vector and $\mathbf{l}$ extracts the displacement at the output degree of freedom. The first constraint $\mathbf{K u}=\mathbf{f}$ refers to the governing equation of linear elasticity, which is solved by finite element analysis. In this equation, $\mathbf{K}$ is the stiffness matrix defined by the SIMP scheme (see Appendix A) and $\mathbf{f}$ denotes the external load vector. The second constraint is on the structural volume, where $\mathbf{v}$ is a vector with elemental volumes, $\bar{\rho}$ is the relative material density vector $(\mathbf{0} \leq \overline{\boldsymbol{\rho}} \leq \mathbf{1})$ and $V^{*}$ is the upper allowable volume.

Note that for practical design of compliant mechanisms it is important to consider geometrical non-linearity [43]. However, since the aim of this paper is not to come up with an actual manufactured piece but rather to propose a general methodology for design of systems to be manufactured by optical microlithography, the exact representation of the physics is not essential here. A practical example would also need determination of the exact lithography parameters for the considered manufacturing setup. The considered linear formulation (16) fully covers all the critical aspects of the procedure applied to compliant mechanism design, such as e.g. formation of thin and fragile hinges when manufacturing variations are not taken into account and the appearance of robust hinges when manufacturing constraints are taken into account. The inclusion of a non-linear formulation would possibly result in different overall topologies and objective values but general tendencies and the critical hinge aspect would remain the same.

\subsection{SMO problem formulation}

According to the discussions in the previous section, the corresponding source and mask optimization problem for the micro-gripper design problem for optical projection lithography is 
given as follows:

$$
\begin{aligned}
\underset{\phi, \boldsymbol{\sigma}}{\min }: & L=E\left\{C_{\eta}^{\beta}\right\}, \\
\text { s.t. : } & C_{\eta}^{\beta}=-\mathbf{l}^{\top} \mathbf{u}_{\eta}^{\beta}, \\
: & \mathbf{K}\left(P_{\eta}^{\beta}\right) \mathbf{u}_{\eta}^{\beta}=\mathbf{f}, \\
: & P_{n}^{\top} \mathbf{v} \leq V^{*}, \\
: & \beta \in\left[\beta_{\min }, \beta_{\max }\right], \eta \in\left[\eta_{\min }, \eta_{\max }\right], \\
: & \phi_{i}=\{0,1\}, \quad i=1: N_{m}, \\
: & 0 \leq \sigma_{j} \leq 1, \quad j=1: N_{s},
\end{aligned}
$$

where $C_{\eta}^{\beta}$ denotes the displacement of the micro-gripper pattern developed at the lens defocus $\beta$ and exposure threshold $\eta$ and $E$ captures its mean value over the variation ranges. The volume constraint is imposed on the nominal pattern $P_{n}$ developed at the ideal manufacturing condition. In order to utilize the sequential optimization scheme, the overall design problem Eq.17 is split into sub-optimization problems for the mask:

$$
\begin{aligned}
\underset{\phi}{\min _{\boldsymbol{\phi}}:} & L=E\left\{C_{\eta}^{\beta}\right\}+\frac{\lambda_{1}}{n_{e}} \sum_{e=1}^{n_{e}} \phi\left(\mathbf{x}_{e}\right)\left(1-\phi\left(\mathbf{x}_{e}\right)\right)+\frac{\lambda_{2}}{n_{e}} \sum_{e=1}^{n_{e}}\left|\nabla \rho\left(\mathbf{x}_{e}\right)\right|^{2}, \\
\text { s.t. : } & C_{\eta}^{\beta}=-\mathbf{l}^{\top} \mathbf{u}_{\eta}^{\beta}, \\
: & \mathbf{K}\left(P_{\eta}^{\beta}\right) \mathbf{u}_{\eta}^{\beta}=\mathbf{f}, \\
: & P_{n}^{\top} \mathbf{v} \leq V^{*}, \\
: & \beta \in\left[\beta_{\min }, \beta_{\max }\right], \eta \in\left[\eta_{\min }, \eta_{\max }\right], \\
: & 0 \leq \phi(\mathbf{x}) \leq 1,
\end{aligned}
$$

and for the illumination source:

$$
\begin{aligned}
\underset{\boldsymbol{\sigma}}{\min .}: & L=E\left\{C_{\eta}^{\beta}\right\} \\
\text { s.t. : } & C_{\eta}^{\beta}=-\mathbf{l}^{\top} \mathbf{u}_{\eta}^{\beta}, \\
: & \mathbf{K}\left(P_{\eta}^{\beta}\right) \mathbf{u}_{\eta}^{\beta}=\mathbf{f}, \\
: & P_{n}^{\top} \mathbf{v} \leq V^{*} \\
: & \beta \in\left[\beta_{\min }, \beta_{\max }\right], \eta \in\left[\eta_{\min }, \eta_{\max }\right], \\
: & 0 \leq \sigma(\mathbf{x}) \leq 1 .
\end{aligned}
$$

These two sub-optimization problems Eqs. (18) and (19) are solved here using the Method of Moving Asymptote [44]. The sensitivity analysis is given in Appendix B.

\subsection{Design results}

An initial mask is conceived by expanding the design domain $\Omega$ with supportive features as shown in Fig. 6(b), which prevent the pattern from being detached off the boundary conditions. The mask has an area of $3 \mu \mathrm{m} \times 3 \mu \mathrm{m}$ and it is discretized into $200 \times 200$ square elements with resolution $15 \mathrm{~nm} \times 15 \mathrm{~nm}$ per element (pixel). The height $1 \mu \mathrm{m}$ is assumed for the final structure. The Young's modulus $E_{0}=60 \mathrm{GPa}$ and a Poisson's ratio $\nu=0.3$ are assumed for the solid material. The upper volume fraction for the nominal pattern developed at $\eta=0.5, \beta=0 \mathrm{~nm}$ is limited to $V^{*}=0.3$. The considered process uncertainty of defocus and exposure dose variations are in the range of $\beta=[-100 \mathrm{~nm}, 100 \mathrm{~nm}]$ and $\eta=[0.4,0.6]$. Other parameters are set as $\lambda=193 \mathrm{~nm}, N A=1.25$, $\alpha=64, \mathrm{f}_{\mathrm{in}}=60 \mu \mathrm{N}, \mathrm{k}_{\mathrm{in}}=0.2 \mathrm{mN} / \mu \mathrm{m}$ and $\mathrm{k}_{\mathrm{out}}=0.005 \mathrm{mN} / \mu \mathrm{m}$. Furthermore, the illumination source is discretized into $100 \times 100$ pixels for computation. In the mask optimization, 15 kernels are chosen as the reduced basis set to calculate the aerial image and a continuation scheme [2] is 


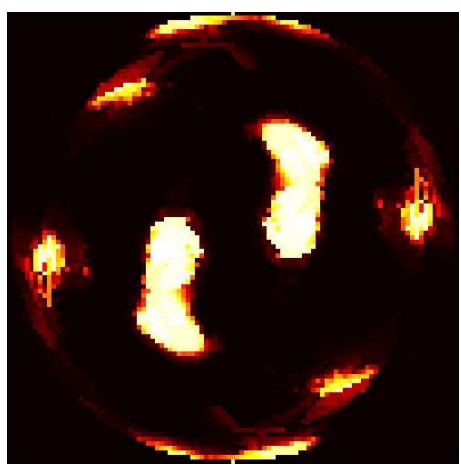

(a) Effective source

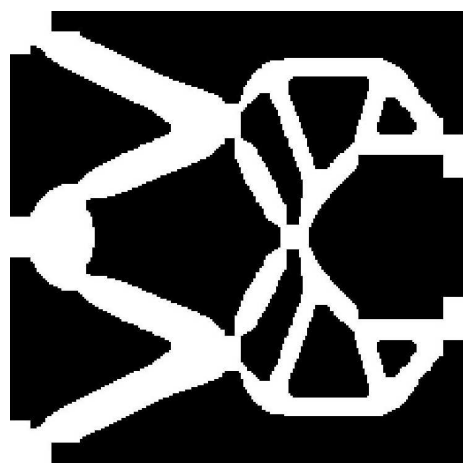

(b) Mask

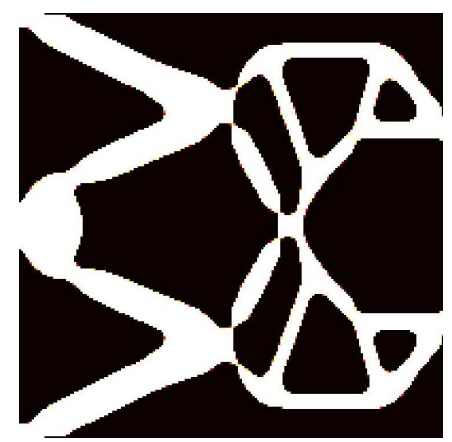

(c) Developed pattern, $C=467 \mathrm{~nm}$

Figure 7. The optimization results without considering manufacturing errors: (a) the optimized source; (b) the optimized mask with supportive features; (c) the developed pattern .

adopted to update $\alpha, \lambda_{1}$ and $\lambda_{2}$. In addition, the PSF for each source point is defined inside the area of $[-300,300] \mathrm{nm} \times[-300,300] \mathrm{nm}$ and its value is assumed vanishing outside.

Fig. 8 records the SMO results for each sub-optimization problem without considering manufacturing errors, where only the displacement of the nominal pattern is considered as the objective. As shown in Fig. 8(b), an optimized mask is first obtained under an annular source of coherent factors 0.4/0.6, which is given in Fig. 8(a). The resulting mechanism shown in Fig. 8(c) exhibits a displacement of $450 \mathrm{~nm}$. Then, by fixing the optimized binary mask, a source is computed as shown in Fig. 8(d), for which the resulting mechanism has a larger displacement of $455 \mathrm{~nm}$, thus a better performance. Moreover, one additional round of SMO is carried out and the optimization results are presented in Figs. 8(g-l). Throughout this sequential design process, the layouts of both the mask and source are continuously changing, while the performance of the mechanism improves from $450 \mathrm{~nm}$ to $468 \mathrm{~nm}$. However, the performance gain slows down after two rounds of SMOs and additional optimization does not show significant improvement. It is interesting to note that although the two illumination sources shown in Figs. 8(g) and (j) are different, the final patterns in Figs. 8(i) and (l) are almost the same in geometry as well as in the mechanism behavior. This phenomenon is attributed to high degrees of non-uniqueness in the design of the source. However, it does not hinder the effectiveness of the proposed solution in finding a pair of mask and source, which can directly yield a micro-device with optimized performance.

One issue regarding the compliant mechanism design as shown in Fig. 8 is the tiny hinges that connecting structural members. Those hinges can easily disappear if the manufacturing conditions fluctuate, in turn destroying the integrity and functionality of the mechanism. To avoid this issue, the robust formulation proposed in the previous section is applied to consider 9 patterns which are developed under the manufacturing conditions $\{(\beta, \eta) \mid \beta=\{-100,0,100\} \mathrm{nm}, \eta=\{0.4,0.5,0.6\}\}$. The weight factor $1 / 9$ for each pattern is used to calculate the mean value $E$ in Eq. (12). Fig. 9 shows the optimized source, mask (with supportive features) and developed patterns after two rounds of 


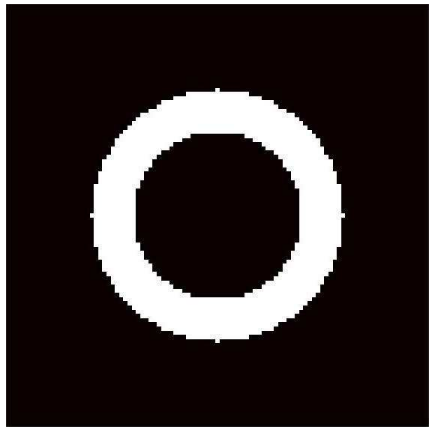

(a) Fixed initial source

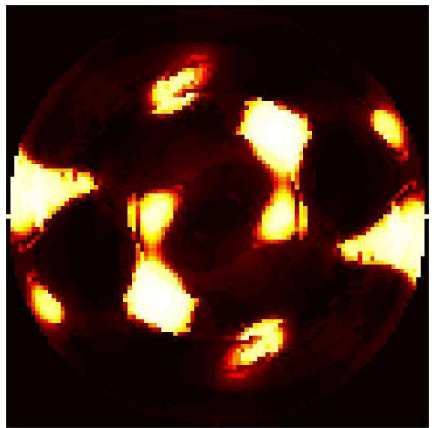

(d) Optimized source I

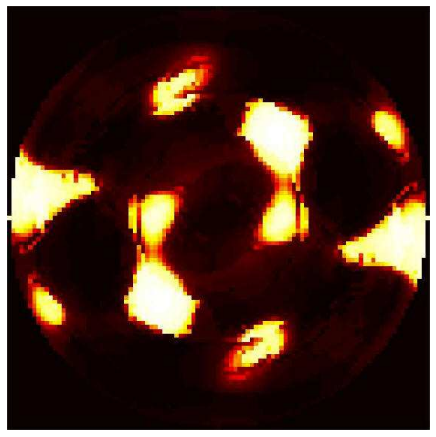

(g) Fixed source I

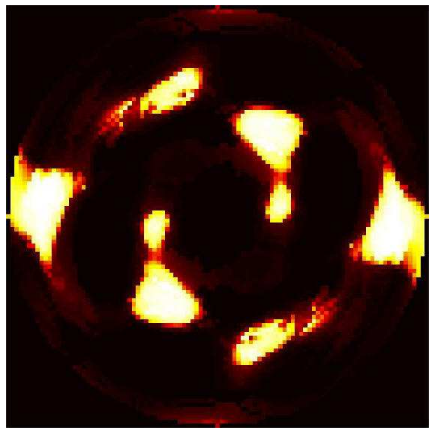

(j) Optimized source II

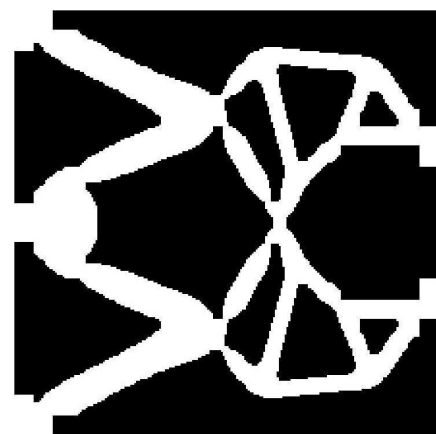

(b) Optimized Mask I

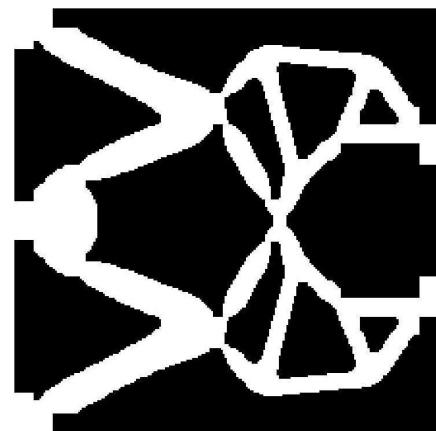

(e) Fixed mask I

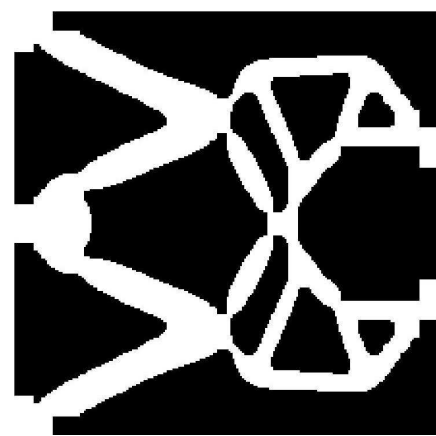

(h) Optimized mask II

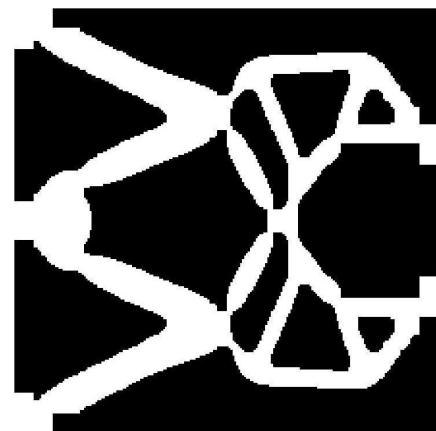

(k) Fixed mask II

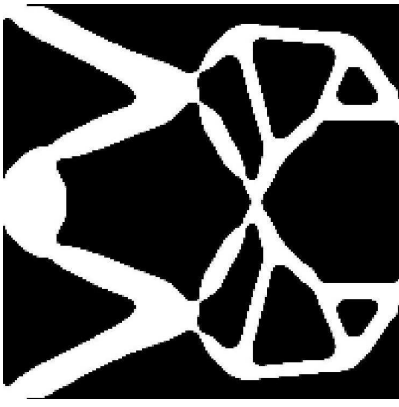

(c) Pattern, $C=450 \mathrm{~nm}$

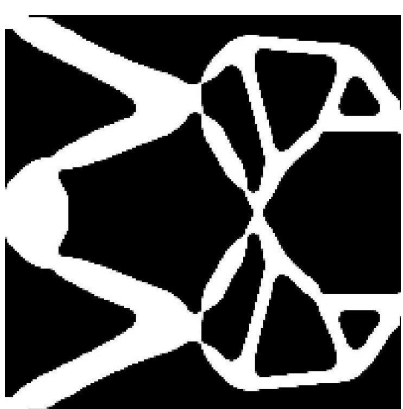

(f) Pattern, $C=455 \mathrm{~nm}$

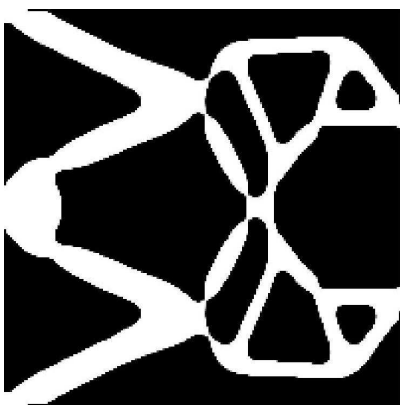

(i) Pattern, $C=467 \mathrm{~nm}$

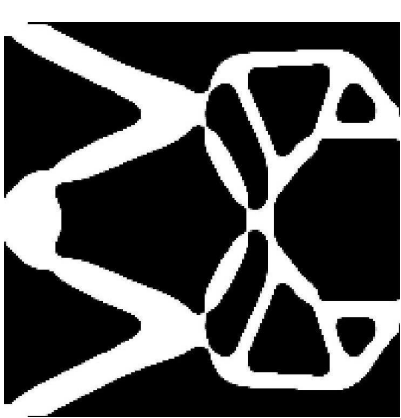

(l) Pattern, $C=468 \mathrm{~nm}$

Figure 8. History of two rounds of SMOs for a compliant micro-gripper without considering manufacturing errors: (column from left to right) source, mask (with supportive features) and the developed pattern at the nominal manufacturing condition $(\eta, \beta)=(0.5,0 \mathrm{~nm})$; (a-c) $1^{\text {st }}$ mask optimization results; (d-f) $1^{\text {st }}$ source optimization results; (g-i) $2^{\text {nd }}$ mask optimization results; (j-1) $2^{\text {nd }}$ source optimization (final) results. It is worth noting that for each sub-optimization, the mechanism pattern possesses tiny hinges. 
SMO. Comparing the nominal patterns between Fig. 8(1) and Fig. 9(g), the latter exhibits a finite minimum length scale seen in the thicker structural joints. The enforcement of minimum length scale and robustness comes at the cost of a smaller displacement of $435 \mathrm{~nm}$. No degraded pattern is observed within the considered variation ranges by using the obtained source and mask as shown in Figs. 9(a-b). All patterns therein achieve displacements between $410 \mathrm{~nm}$ to $445 \mathrm{~nm}$ and the mean value is $431 \mathrm{~nm}$.

\section{DISCUSSIONS AND FUTURE WORK}

An optimum design solution for micro-manufacturing is presented to design source and mask design for optical microlithography with partially coherent illumination. The solution unifies topology optimization of mechanical structures and computational lithography based on the premises that both computational approaches share a similar parameterization and workflow. It combines the advantages in both technologies that the optimized design performance and manufacturability are guaranteed at the same time. A compliant micro-gripper design example is presented to demonstrate the applicability of the approach, but it can equally well be extended to other design problems with different objectives and constraints.

The idea has been successfully realized for microlithography with a single exposure resolution enhancement technique. It is worthwhile to investigate multiple-patterning lithography in order to push the limit of the feature size below $40 \mathrm{~nm}$ with $193 \mathrm{~nm}$ photolithography [9]. Besides, combination with self-assembly and next-generation of micro- / nano- manufacturing technologies may be subject to future work. The suggested solution framework can be further extended by well-established techniques from both fields of multidisciplinary topology optimization and computational lithography. One extension is to incorporate the recent work by [11] that considers polarization angle of the source as an additional design variable to enable more design freedom in PEC. In addition, various regularization approaches [39, 38, 22] can be applied to control the final mask and source to meet actual manufacturing and utility requirements. It would also be interesting to study feature-control approaches [45] in the proposed solution to design micro- / nano- devices with desirable length scales.

\section{APPENDIX A: SOLID ISOTROPIC MATERIAL INTERPOLATION (SIMP)}

According to the Solid Isotropic Material interpolation with Penalization (SIMP) scheme [26], the element stiffness $\mathbf{K}_{e}$ is modeled as:

$$
\mathbf{K}_{e}=\left(E_{\min }+\overline{\boldsymbol{\rho}}_{e}^{p}\left(E_{0}-E_{\min }\right)\right) \mathbf{K}_{0},
$$

where $\mathbf{K}_{0}$ denotes the element stiffness matrix for unit stiffness, $E_{0}$ the Young's modulus of the material phase, $\bar{\rho}$ is the element density and $p$ the penalization power. For the numerical example in this paper, $E_{\min }=10^{-9} E_{0}$ is set to avoid numerical singularity in finite element analysis and the penalization power $p=3$ is used.

\section{APPENDIX B: SENSITIVITY ANALYSIS}

In the mask optimization, because the reduced-order approach Eq. (14) is used to compute the aerial image, the sensitivity of an objective functional $C$ w.r.t. the mask $\phi$ is written by:

$$
\begin{gathered}
\frac{\partial C}{\partial \phi}=2 \cdot \sum_{i=1}^{M s} \operatorname{Re}\left(\overline{G_{i}^{*}} \otimes\left(\frac{\partial F}{\partial P} \cdot \frac{\partial P}{\partial A} \cdot\left(G_{i} \otimes \boldsymbol{\phi}\right)\right)\right), \\
\frac{\partial P}{\partial A}=\frac{\alpha \cdot\left(1-\tanh ^{2}(\alpha \cdot(A(\mathbf{x})-\eta))\right.}{\tanh (\alpha \cdot \eta)+\tanh (\alpha \cdot(1.0-\eta))}
\end{gathered}
$$




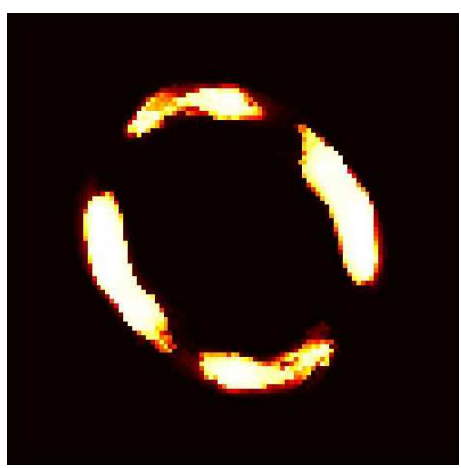

(a) Effective source

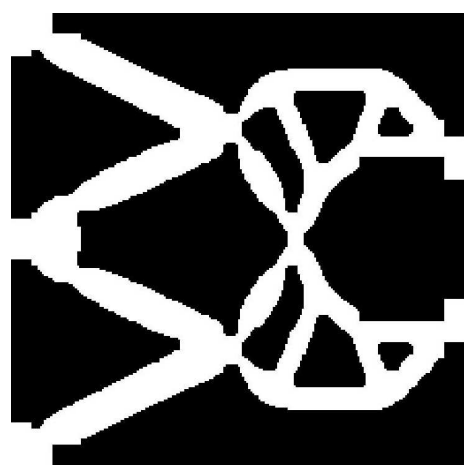

(b) Mask

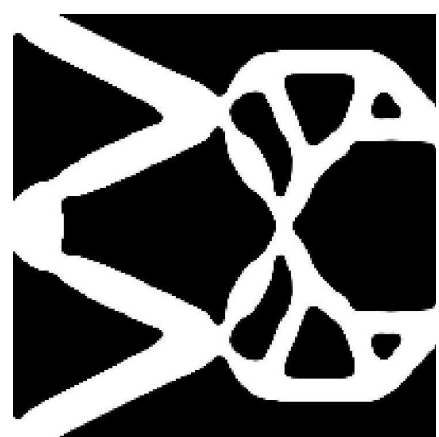

(c) $(0.35,100 \mathrm{~nm}), C=410 \mathrm{~nm}$

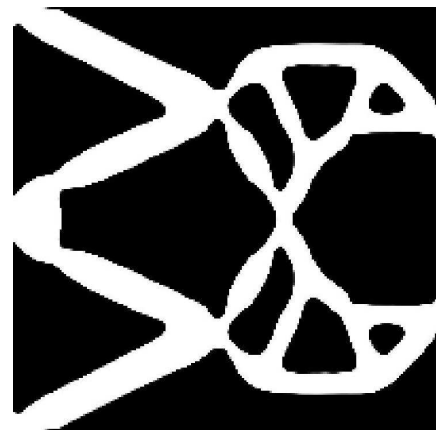

(f) $(0.35,0 \mathrm{~nm}), C=417 \mathrm{~nm}$

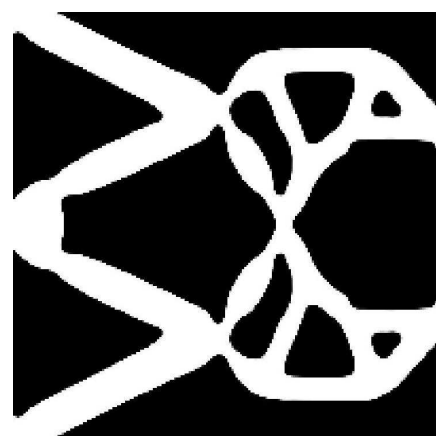

(i) $(0.35,-100 \mathrm{~nm}), C=411 \mathrm{~nm}$

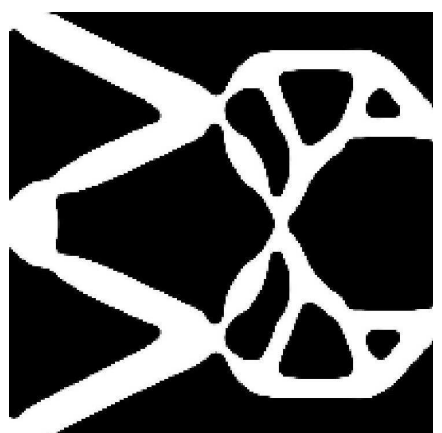

(d) $(0.5,100 \mathrm{~nm}), C=438 \mathrm{~nm}$

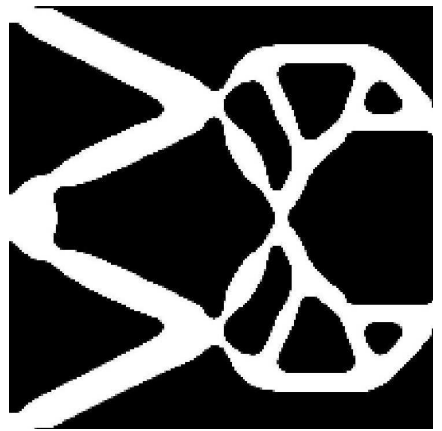

(g) $(0.5,0 \mathrm{~nm}), C=435 \mathrm{~nm}$

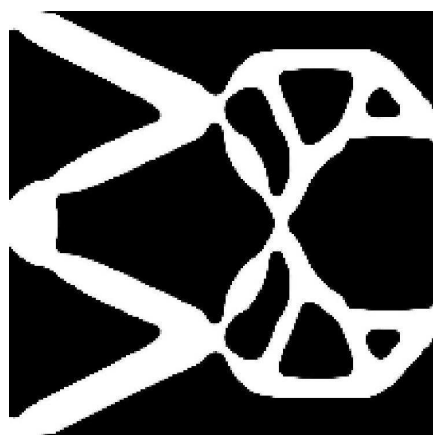

(j) $(0.5,-100 \mathrm{~nm}), C=439 \mathrm{~nm}$

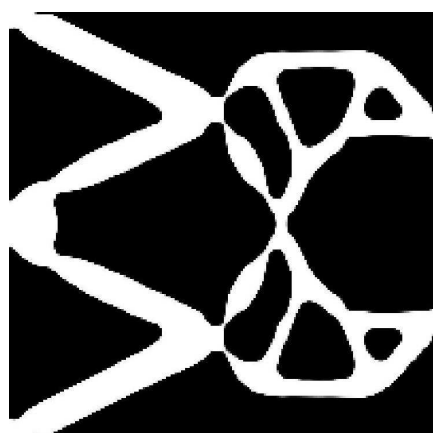

(e) $(0.65,100 \mathrm{~nm}), C=445 \mathrm{~nm}$

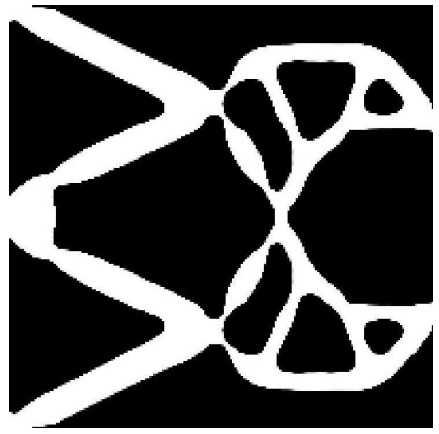

(h) $(0.65,0 \mathrm{~nm}), C=439 \mathrm{~nm}$

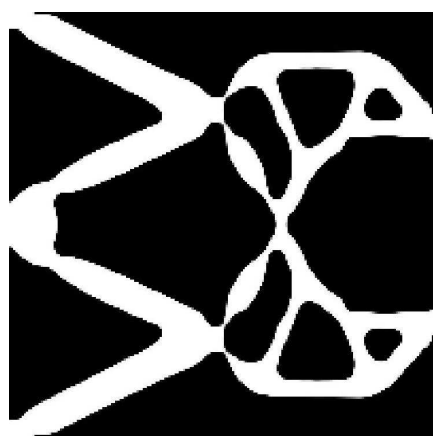

(k) $(0.65,-100 \mathrm{~nm}), \mathrm{C}=443 \mathrm{~nm}$

Figure 9. The optimization results with considering manufacturing errors: (a) the optimized source; (b)the optimized mask with supportive features; (c-k) the developed patterns at different manufacturing conditions $(\eta, \beta)$. 
where the symbol $\operatorname{Re}$ denotes the real part of a complex number, $G^{*}$ is the complex conjugate of $G$ and $\bar{G}$ rotates the matrix $G$ by $180^{\circ}$.

For the source optimization with the full Abbe's model, the sensitivity of $C$ w.r.t the source point $\sigma_{i}$ is given as:

$$
\frac{\partial C}{\partial \sigma_{i}}=\left[\frac{\partial F}{\partial P} \cdot \frac{\partial P}{\partial A} \cdot \frac{\left[\left(\boldsymbol{\phi} \otimes H_{i}\right)(\mathbf{x})\right]^{2}-A\left\|\Sigma_{\mathbf{x}} H_{i}(\mathbf{x})\right\|^{2}}{\Sigma_{i=1}^{N s} \sigma_{i}\left\|\Sigma_{\mathbf{x}} H_{i}(\mathbf{x})\right\|^{2}}\right]
$$

\section{ACKNOWLEDGEMENT}

The authors acknowledge the financial support received from the Hans Christian Ørsted Postdoctoral grant at the Technical University of Denmark, from the EU research project "LaScISO" (Grant No. 285782), from the Villum Foundation (the NextTop project) and from the research project "Sapere Aude TOpTEn" (Topology Optimization of Thermal ENergy systems) from the Danish Council for Independent Research, grant: DFF4005-00320. The authors thank Dr. Erik Andreassen, Dr. Haojie Lian and Mr. Christian Lundgaard from the TopOpt group of the Technical University of Denmark for valuable discussions.

\section{REFERENCES}

1. Jansen M, Lazarov BS, Schevenels M, Sigmund O. On the similarities between micro/nano lithography and topology optimization projection methods. Struct. Multidiscip. Optim. 2013; 48:717-730.

2. Zhou M, Lazarov B, Sigmund O. Topology optimization for optical projection lithography with manufacturing uncertainties. Applied Optics 2014; 53:2720-2729.

3. Wong AK. Resolution enhancement techniques in optical lithography. SPIE, 2001.

4. Lee SY. A flexible and efficient approach to e-beam proximity effect correctionłpyramid. Surface and Interface Analysis, Proc. of NIST workshop on Modeling Electron Transport for Applications in Electron and X-Ray Analysis and Metrology 2005; 37(11):919-926.

5. Peckerar M, Chang S, Marrian C. Proximity correction algorithms and a co-processor based on regularized optimization. i: Description of the algorithm. J Vac Sci Technol B 1995; 13(6)::2518-2525.

6. Peckerar M, Sander D, Srivastava A, Foli A, U UV. Electron beam and optical proximity effect reduction for nanolithography: new results. J Vac Sci Technol B 2007; 25(6)::2288-2294.

7. Dobisz E, Bandić Z, Peckerar M. Electron beam nanolithography. In: Microlithography: Science and Technology, Second Edition, CH. 15, CRC Press 2007; :799-836.

8. Mack C. Fundamental principles of optical lithography: the science of micro-fabrication. Wiley, 2007.

9. Pan DZ, Yu B. Design for manufacturing with emerging nanolithography. Computer-Aided Design of Integrated Circuits and Systems, IEEE Transactions on 2013; 32(10)::1453-1472.

10. Poonawala A, Milanfar P. Mask design for optical microlithography an inverse imaging problem. IEEE Transactions on Image Processing 2007; 16(3):774-788.

11. Ma X, Gao J, Han C, Li Y, Dong L, Liu L. Efficient source polarization optimization for robust optical lithography. Proc. SPIE 9052, Optical Microlithography XXVII, 90520T 2014;

12. Lv W, Liu S, Wu X, Lam EY. Illumination source optimization in optical lithography via derivative-free optimization. J. Opt. Soc. Am. A 2014; 31(12):B19-B26.

13. Ma X, Arce GR. Generalized inverse lithography methods for phase-shifting mask design. Optics Express 2007; 15(23):15 066-15079.

14. Rosenbluth AE, Bukofsky S, Fonseca C, Hibbs M, Lai K, Molless AF, Singh RN, Wong AKK. Optimum mask and source patterns to print a given shape. J. Microlith. Microfab. Microsyst. 2002; 1(1):13-30.

15. Ma X, Arce GR. Pixel-based simultaneous source and mask optimization for resolution enhancement in optical lithography. Opt. Express 2009; 17(7):5783-5793.

16. Peng Y, Zhang J, Wang Y, Yu Z. Gradient-based source and mask optimization in optical lithography. IEEE Trans. Image Process. 2011; 20:2856-2864.

17. Yu J, Yu P. Gradient-based fast source mask optimization (smo). Proc. SPIE 7973, 2011; 797320.

18. Jia N, Lam EY. Pixelated source mask optimization for process robustness in optical lithography. Opt. Express 2011; 19:19384-19398.

19. J Li YS, Lam E. Hotspot-aware fast source and mask optimization. Opt. Express 2012; 20(19):21 792-21 804.

20. J Li SL, Lam E. Efficient source and mask optimization with augumented lagrangian methods in optical lithography. Opt. Express 2013; 21(7):8076-8090.

21. S Li XW, Bu Y. Robust pixel-based source and mask optimization for inverse lithography. Opt. Laser Technol. 2013; 45:285-293.

22. Ma X, Han C, Li Y, Dong L, Arce GR. Pixelated source and mask optimization for immersion lithography. J. Opt. Soc. Am. A 2013; 30(1):112-123.

23. Ma X, Dong L, Han C, Gao J, Li Y, Arce GR. Gradient-based joint source polarization mask optimization for optical lithography. J. Micro/Nanolith. MEMS MOEMS. 2015; 14:023 504.

$\S$ The matrix rotation is due to fact that one Fourier transform (light diffraction) followed by a second one (focusing by the projection lens) produces an image that is $180^{\circ}$ rotated version of the original [8]. 
24. Mulder M, Engelen A, et al. Performance of flexray: a fully programmable illumination system for generation of freeform sources on high-na immersion systems. Proc. SPIE 7640, 2010; 76401P.

25. Bendsøe M, Kikuchi N. Generating optimal topologies in structural design using a homogenization method. Comput Method Appl M 1988; 71:197-224.

26. Bendsøe M, Sigmund O. Topology Optimization - Theory, Methods and Applications. Spinger, 2003.

27. Sigmund O. On the design of compliant mechanisms using topology optimization. Mech. Struct. Machines 1997; 25:493-524.

28. Frandsen LH, Elesin Y, Frellsen LF, Mitrovic M, Ding Y, Sigmund O, Yvind K. Topology optimized mode conversion in a photonic crystal waveguide fabricated in silicon-on-insulator material. Optics Express 2014; 22(7):8525-8532.

29. Guest J, Prevost J, Belytschko T. Achieving minimum length scale in topology optimization using nodal design variables and projection functions. Int. J. Numer. Methods Eng. 2004; 61:238-254.

30. Sigmund O. Morphology-based black and white filters for topology optimization. Struct. Multidiscip. Optim. 2007; 33:401-424.

31. Xu S, Cai Y, Cheng G. Volume preserving nonlinear density filter based on heaviside functions. Structural and Multidisciplinary Optimization 2010; 41(4):495-505.

32. Abbe E. Beiträge zur theorie des mikroskops und der mikroskopischen wahrnehmung. Archiv für mikroskopische Anatomie 1873; 9(1):413-468.

33. Choy SK, Jia N, Tong CS, Tang ML, Lam EY. A robust computational algorithm for inverse photomask synthesis in optical projection lithography. SIAM J. IMAGING SCIENCES 2012; 5(2):625-651.

34. Jia N, Lam EY. Machine learning for inverse lithography: Using stochastic gradient descent for robust photomask synthesis. J. of Optics 2010; 12(4):045 601.

35. Shen Y, Jia N, Wong N, Lam EY. Robust levelset-based inverse lithography. Optics Express 2011; 19(6):5511-5521.

36. Lazarov BS, Schevenels M, Sigmund O. Topology optimization considering material and geometric uncertainties using stochastic collocation methods. Structural and Multidisciplinary Optimization 2012; 46:597-612.

37. Wang F, Lazarov BS, Sigmund O. On projection methods, convergence and robust formulations in topology optimization. Structural and Multidisciplinary Optimization 2011; 43:767-784.

38. Jia N, Lam EY. Performance analysis of pixelated source-mask optimization for optical microlithography. in IEEE International Conference on Electron Devices and Solid-State Circuits, 2010.

39. Poonawala A, Milanfar P. Mask design for optical microlithography an inverse imaging problem. IEEE Trans. Image Process. 2007; 16:774-788.

40. Bourdin B. Filters in topology optimization. Int. J. Numer. Meth. Engng. 2001; 50:2143-2158.

41. Chang SJ, Chen C. Abbe-pca-smo: Microlithography source and mask optimization based on abbe-pca. in Proc. SPIE 7640, Optical Microlithography XXIII, 2010; 764026.

42. Sardan O, Eichhorn V, Petersen D, Fatikow S, Sigmund O, Bøggild P. Rapid prototyping of nanotube-based devices using topology-optimized microgrippers. Nanotechnology 2008; 19:495-503.

43. Pedersen CBW, Buhl T, Sigmund O. Topology synthesis of large-displacement compliant mechanisms. Int. J. Numer. Meth. Engng. 2001; 50:2683-2705.

44. Svanberg K. The method of moving asymptotesła new method for structural optimization. Int. J. Numer. Methods Eng. 1987; 24:359-397.

45. Zhou M, Lazarov BS, Wang F, Sigmund O. Minimum length scale in topology optimization by geometric constraints. Computer Methods in Applied Mechanics and Engineering 2015; 293:266-282. 